\title{
Prospective Study Demonstrates Utility of EP-QuIC in Creutzfeldt-Jakob Disease Diagnoses
}

\author{
Sharon L. R. Simon, Anne Peterson, Clark Phillipson, Jonathan M. Walker, \\ Meika Richmond, Gerard H. Jansen (1D, J. David Knox
}

\begin{abstract}
Prospectively acquired Canadian cerebrospinal fluid samples were used to assess the performance characteristics of three ante-mortem tests commonly used to support diagnoses of Creutzfeldt-Jakob disease. The utility of the end-point quaking-induced conversion assay as a test for Creutzfeldt-Jakob disease diagnoses was compared to that of immunoassays designed to detect increased amounts of the surrogate markers 14-3-3 $\gamma$ and hTau. The positive predictive values of the end-point quaking-induced conversion, 14-3-3 $\gamma$, and hTau tests conducted at the Prion Diseases Section of the Public Health Agency of Canada were 96\%, 68\%, and 66\%, respectively.

RÉSUMÉ : Une étude prospective démontre l'utilité du test EP-QuIC dans le diagnostic de la maladie de Creutzfeldt-Jakob. Des échantillons de liquide cérébrospinal (LCS) obtenus de manière prospective auprès de sujets canadiens ont été utilisés pour évaluer la performance de trois tests ante mortem couramment utilisés pour obtenir des diagnostics de maladie de Creutzfeldt-Jakob (MCJ). À cet effet, l'utilité du test dit de « conversion provoquée par tremblement au point final» (end-point quaking induced conversion ou EP-QuIC) a été comparée à celle de tests immunologiques conçus pour détecter une augmentation du nombre de marqueurs de substitution 14-3-3 $\gamma$ et hTau. Ainsi, les valeurs prédictives positives du test EP-QuIC et de ces deux autres tests immunologiques réalisés par la Section des maladies à prions de l'Agence de la santé publique du Canada ont été respectivement de $96 \%$, $68 \%$ et $66 \%$.
\end{abstract}

Keywords: Creutzfeldt-Jakob disease, Prion, RT-QuIC, EP-QuIC

doi:10.1017/cjn.2020.139

Can J Neurol Sci. 2021; 48: 127-129

Sub-acute spongiform encephalopathies, including CreutzfeldtJakob disease (CJD), constitute a large and heterogeneous group of rare invariably fatal diseases, also known as prion diseases. They occur most often in man as sporadic disease but can also occur as genetic, or infectiously acquired disease. Like most neurodegenerative diseases, prion diseases are caused by disease-associated misfolded proteins. In prion diseases, the misfolded isoform (PrPd) of the host prion protein $(\mathrm{PrPc})^{1}$ is the causative agent, and in instances of prion disease transmission, the transmissible agent is PrPd. This key biological fact precludes the use of conventional technologies, such as polymerase chain reaction and serology, which are commonly applied to the direct and specific detection of infectious agents. As a result, ante-mortem diagnosis of CJD has relied upon clinical presentation, neurological examination, and supporting investigations such as magnetic resonance imaging (MRI) scanning of the brain and cerebrospinal fluid (CSF) testing for the presence of increased amounts of indirect protein markers. The most commonly used markers are $14-3-3^{2}$ and microtubuleassociated $\mathrm{tau}^{3}$. Although neither of these indirect markers is fully diagnostic, both have useful sensitivities and specificities for $\mathrm{CJD}^{4}$.

More recently, a new test, known as real-time quaking-induced conversion (RT-QuIC), demonstrated how the pathogenic protein, PrPd, could be detected more directly ${ }^{5}$. To allow for robust clinical testing, the Prion Diseases Section adapted the RT-QuIC test for use in a diagnostic setting. We call this version of the test end-point quaking-induced conversion (EP-QuIC) ${ }^{6}$. Both RT-QuIC and EP-QuIC exploit the natural ability of the disease-associated, misfolded isoform, PrPd, to induce conversion of the normal cellular form of the prion protein $(\mathrm{PrPc})$ into a misfolded form in vitro.

The Public Health Agency of Canada's Prion Diseases Section (PDS) offers an ante-mortem CSF test panel for CJD consisting of detection of the disease-associated form of the prion protein by EP-QuIC and immunoassays for 14-3-3 $\gamma$ and total tau proteins. In addition, sequence analysis of the prion gene (PRNP) can be performed if blood or other tissues are available. All assays are accredited under Can-P4E (ISO/IEC 17025) and are offered free of charge to Canadian health care providers. The CSF tests can be requested by sending samples directly to the PDS at the National Microbiology Laboratory; a situation fairly unique compared to other international CJDSS surveillance systems.

In this study, 716 prospectively acquired Canadian CSF samples were submitted to the PDS between April 2017 and June 2019. Samples that were subsequently excluded from this study included: 26 samples where insufficient sample quantity was provided by the submitting laboratory; 31 samples that were xanthrochromic or bloody making them unsuitable for testing; 30

From the Prion Diseases Section, National Microbiology Laboratory, Public Health Agency of Canada, Winnipeg, Manitoba, Canada (SLRS, AP, CP, JMW, MR, JDK); and Division of Anatomical Pathology, University of Ottawa, Ottawa, Ontario, Canada (GHJ)

Received April 2, 2020. Final Revisions Submitted June 16, 2020. Date of ACCEPTANCE June 28, 2020.

Correspondence to: J. David Knox, Prion Diseases Section, National Microbiology Laboratory, Public Health Agency of Canada, 1015 Arlington St, Winnipeg, Manitoba MB R3E 3R2, Canada. Email: david.knox2@canada.ca 
Table 1: Comparison of the performance characteristics of EP-QuIC, 14-3-3, and tau

\begin{tabular}{|c|c|c|c|c|c|c|c|c|}
\hline & \multicolumn{2}{|c|}{ CJD } & \multicolumn{2}{|c|}{ Non-CJD } & \multirow{2}{*}{$\begin{array}{c}\text { Sensitivity } \\
a /(a+b)\end{array}$} & \multirow{2}{*}{$\begin{array}{c}\text { Specificity } \\
d /(d+c)\end{array}$} & \multirow{2}{*}{$\begin{array}{c}\begin{array}{c}\text { Positive } \\
\text { predictive value }\end{array} \\
a /(a+c)\end{array}$} & \multirow{2}{*}{$\begin{array}{c}\begin{array}{c}\text { Negative } \\
\text { predictive value }\end{array} \\
d /(d+b) \\
\end{array}$} \\
\hline & True positive (a) & $\begin{array}{c}\text { False } \\
\text { negative (b) }\end{array}$ & $\begin{array}{c}\text { False } \\
\text { positive (c) }\end{array}$ & $\begin{array}{c}\text { True } \\
\text { Negative (d) }\end{array}$ & & & & \\
\hline \multirow[t]{2}{*}{ EP-QuiC } & \multirow[t]{2}{*}{117} & \multirow[t]{2}{*}{5} & \multirow[t]{2}{*}{5} & \multirow[t]{2}{*}{496} & $96 \%$ & $99 \%$ & $96 \%$ & $99 \%$ \\
\hline & & & & & $90.7-98.7$ & $97.7-99.7$ & $90.7-98.3$ & $97.7-99.6$ \\
\hline \multirow[t]{2}{*}{$14-3-3$} & \multirow[t]{2}{*}{103} & \multirow[t]{2}{*}{19} & \multirow[t]{2}{*}{49} & \multirow[t]{2}{*}{452} & $84 \%$ & $90 \%$ & $68 \%$ & $96 \%$ \\
\hline & & & & & $76.8-90.4$ & $87.3-92.7$ & $61.5-73.5$ & $94.0-97.3$ \\
\hline \multirow[t]{2}{*}{ Tau } & \multirow[t]{2}{*}{112} & \multirow[t]{2}{*}{10} & \multirow[t]{2}{*}{59} & \multirow[t]{2}{*}{442} & $92 \%$ & $88 \%$ & $66 \%$ & $98 \%$ \\
\hline & & & & & $85.4-96.0$ & $85.1-90.9$ & $59.8-70.8$ & $96.1-98.8$ \\
\hline
\end{tabular}

Table 2: Detailed analyses of false positive and false negative results

\begin{tabular}{|c|c|c|c|c|c|c|c|}
\hline \multirow[b]{2}{*}{ Sample } & \multirow[b]{2}{*}{ Diagnoses } & \multirow[b]{2}{*}{ Mutation } & \multirow[b]{2}{*}{ Codon 129} & \multirow[b]{2}{*}{$14-3-3$} & \multirow[b]{2}{*}{ Tau } & \multicolumn{2}{|c|}{ QuIC Assay } \\
\hline & & & & & & $\begin{array}{l}\text { Commercial } \\
\text { substrate }\end{array}$ & In-house substrate \\
\hline 1 & Definite CJD & $\mathrm{n} / \mathrm{a}$ & $\mathrm{V} / \mathrm{V}$ & $\mathrm{TP}$ & $\mathrm{TP}$ & $\mathrm{n} / \mathrm{d}$ & FN \\
\hline 2 & Probable CJD & $\mathrm{D} 178 \mathrm{~N}$ & $\mathrm{M} / \mathrm{M}$ & $\mathrm{FN}$ & FN & $\mathrm{n} / \mathrm{d}$ & FN \\
\hline 3 & Definite CJD & no mutation & $\mathrm{V} / \mathrm{V}$ & $\mathrm{TP}$ & $\mathrm{TP}$ & FN & $\mathrm{TP}$ \\
\hline 4 & Definite CJD & $\mathrm{n} / \mathrm{a}$ & $\mathrm{M} / \mathrm{M}$ & $\mathrm{TP}$ & $\mathrm{TP}$ & FN & $\mathrm{TP}$ \\
\hline 5 & Probable CJD & no mutation & $\mathrm{M} / \mathrm{M}$ & FN & $\mathrm{TP}$ & FN & FN \\
\hline 6 & $\begin{array}{l}\text { Autoimmune } \\
\text { encephalopathy }\end{array}$ & $\mathrm{n} / \mathrm{a}$ & $\mathrm{n} / \mathrm{a}$ & $\mathrm{TN}$ & $\mathrm{TN}$ & $\mathrm{n} / \mathrm{d}$ & FP \\
\hline 7 & $\begin{array}{l}\text { Unknown, but not } \\
\text { suspected CJD }\end{array}$ & $\mathrm{n} / \mathrm{a}$ & $\mathrm{n} / \mathrm{a}$ & $\mathrm{TN}$ & $\mathrm{TN}$ & $\mathrm{n} / \mathrm{d}$ & FP \\
\hline 8 & $\begin{array}{l}\text { Autoimmune } \\
\text { encephalopathy }\end{array}$ & no mutation & $\mathrm{M} / \mathrm{V}$ & FP & FP & FP & $\mathrm{TN}$ \\
\hline 9 & $\begin{array}{l}\text { Unknown, but not } \\
\text { suspected CJD }\end{array}$ & $\mathrm{n} / \mathrm{a}$ & $\mathrm{n} / \mathrm{a}$ & $\mathrm{TN}$ & $\mathrm{TN}$ & FP & $\mathrm{TN}$ \\
\hline 10 & $\begin{array}{l}\text { Unknown, but not } \\
\text { suspected CJD }\end{array}$ & $\mathrm{n} / \mathrm{a}$ & $\mathrm{n} / \mathrm{a}$ & $\mathrm{TN}$ & $\mathrm{TN}$ & FP & $\mathrm{n} / \mathrm{d}$ \\
\hline
\end{tabular}

$\mathrm{FN}=$ false negative; $\mathrm{FP}=$ false positive $\mathrm{TN}=$ true negative; $\mathrm{TP}=$ true positive.

samples where diagnosis is still pending and six samples with indeterminate QuIC results. Xanthrochromic or bloody samples can result in false positive 14-3-3 $\gamma$ and Tau results and false negative EP-QuIC results. In instances where one of the three assay replicates for a QuIC test was positive, the sample was reanalyzed using three different volumes of CSF in the reaction mixture $(7.5,15$, and $30 \mu \mathrm{l})$. Samples consistently exhibiting one of the three assay replicate wells as positive were scored as indeterminate.

Upon release of the CSF testing results, clinicians are directly contacted by phone by the Canadian Creutzfeldt-Jakob Disease Surveillance System (CJDSS). During these conversations, the level of clinical suspicion is discussed. In cases of positive test results and cases of unexpected negative test results, the clinician will be asked to request verbal consent from the patient or their representative to enroll in the CJDSS. Where consent is received, there is periodical follow-up with chart review, MRI and electroencephalogram review, and/or eventual autopsy. In many cases of clinically expected negative test results, an alternative diagnosis is obtained from the clinician, but in an equally significant number no diagnosis is given. The latter leads to a classification as "unknown, but not suspected CJD." As such the patient group of the 623 samples included: 98 autopsy confirmed definite, and 24 probable CJD cases (classified according to the WHO and European Creutzfeldt-Jakob Disease Surveillance Network criteria ${ }^{7}$ ); 222 with alternative diagnoses (Supplementary Table 1); and 279 unknown, but not suspected CJD. Two of the probable samples were drawn from the same patient at two time points separated by $40 \mathrm{~d}$. The raw data as well as the calculated sensitivities and specificities of the three tests are shown in Table 1. The EP-QuIC results are comparable to the RT-QuIC test results reported elsewhere ${ }^{8}$. The relative performance of the three markers for CJD diagnoses is most clearly demonstrated by a calculation of the positive predictive values (Table 1).

A further investigation of the five false negative and five false positive results obtained by EP-QuIC analyses identified two confounding factors that may in part explain these erroneous results. The key component of QuIC analyses is the recombinant 
PrP (rPrP) used as substrate in QuIC assays. At one point during this study, we switched from using our in-house-generated rPrP to a commercially available substrate that was used in the analyses of 215 of the samples reported. Part of the impetus for making this change was to reduce the workload of technicians. Instead, when introduced into the diagnostic workflow, the commercial substrate had exactly the opposite effect due to the number of failed runs that required repeating. We now exclusively use in-house substrate for all our diagnostic EP-QuIC runs. An annual quality review revealed that $3 / 5$ false negative and $3 / 5$ false positive samples were run with commercial substrate. Retrospective reanalysis of five of these ten false samples was performed using in-housegenerated substrate. This correctly resolved the two reanalysed false positive results as well as $2 / 3$ false negative results obtained previously. The characteristics of the ten false positive and negative samples with respect to their initial EP-QuIC results, including the four samples that were correctly resolved when run with inhouse substrate, are presented in Table 2.

The second possible confounding factor is that differences arising from point mutations or insertion/deletions in the prion gene sequence, $P R N P$, may result in a PrPc amino acid sequence more or less likely to induce the conversion of the rPrP substrate. Investigation of this factor occurs through genetic sequence analysis. A prerequisite for the performance of this type of analysis is the receipt of informed consent from the patient or their legal representative. Genetic analyses were performed on 84 patients of whom CSF samples were included in this study. Twelve point mutations, two deletions, and one insertion were observed (Supplementary Table 2). EP-QuIC analyses of the 15 samples associated with mutations produced one false negative result. The CJD causative mutation in this incorrectly identified sample was D178N with homozygous methionine at the polymorphic codon 129. The patient exhibited weight loss and sleep disturbances characteristic of the disease caused by this genetic profile, known as Fatal Familial Insomnia (FFI). In addition to this particular FFI sample, three other FFI samples have been analyzed at our facility. Two of these three FFI samples were also classified as false negatives by EP-QuIC. The possible presence of genetic CJD varieties that are not well detected by EP-QuIC highlights the importance of concurrent genetic testing of patients regardless of the EP-QuIC results. This would enable the detection of genetic CJD cases with a false negative EP-QuIC result.

The important contributions of substrate quality and complementary sequencing to the confirmation of suspected cases of CJD are evident in these results ${ }^{9}$. This is underscored by the inclusion of EP-QuIC in the Canadian national case definition of CJD, where a positive EP-QuIC assay in the presence of a rapidly progressive neurological syndrome and insufficient evidence of an alternative diagnosis is classified as a probable CJD case.

Additional testing, such as an autopsy when patients have passed away, can help in establishing additional diagnostic certainty and provide help in identifying the subtype of prion disease in each patient.

Further information regarding test parameters as well as requisition forms and shipping instructions can be found in our website (https://cnphi.canada.ca). Reporting suspected cases to the CJDSS (1-888-489-2999) and local/provincial public health authority is strongly advised.

\section{ACKNOWLEDGMENTS}

We thank the patients and their families for their participation and generosity, without which we would be unable to perform this work. We thank Tim Connolly of the CJDSS, Centre for Foodborne, Environmental and Zoonotic Infectious Diseases, Public Health Agency of Canada for his assistance in collating the results.

\section{FUNDING}

This research was supported by the Public Health Agency of Canada.

\section{Disclosures}

The authors have no conflicts of interest to declare.

\section{Statement of Authorship}

SLRS: collation of data, writing review, and editing. AP: performance of diagnostic assays, data review, and editing. CP, JMW, and MR: performance of diagnostic assays. GHJ: performance of pathological analyses and review. JDK: study design, data analysis, and drafting of manuscript.

\section{Supplementary Material}

To view supplementary material for this article, please visit https://doi.org/10.1017/cjn.2020.139.

\section{REFERENCES}

1. Ironside JW, Ritchie DL, Head MW. Prion diseases. Handb Clin Neurol. 2017;145:393-403.

2. Hsich G, Kenney K, Gibbs CJ, et al. The 14-3-3 brain protein in cerebrospinal fluid as a marker for transmissible spongiform encephalopathies. N Engl J Med. 1996;335(13):924-30.

3. Otto M, Wiltfang J, Tumani $\mathrm{H}$, et al. Elevated levels of tau-protein in cerebrospinal fluid of patients with Creutzfeldt-Jakob disease. Neurosci Lett. 1997;225(3):210-12.

4. Coulthart MB, Jansen GH, Cashman N. Interpretation of cerebrospinal fluid protein tests in the diagnosis of sporadic CreutzfeldtJakob disease: an evidence-based approach. CMAJ 2014;186: E333-9,

5. Orrù $\mathrm{CD}$, Groveman $\mathrm{BR}$, Hughson $\mathrm{AG}$, et al. RT-QuIC assays for prion disease detection and diagnostics. Methods Mol Biol. 2017; 1658:185-203

6. Cheng K, Vendramelli R, Sloan A, et al. End-point quaking-induced conversion (EP-QuIC): a sensitive, specific, and high-throughput method for the ante-mortem diagnosis of Creutzfeldt-Jacob Disease.): a sensitive, specific, and high-throughput method for the ante-mortem diagnosis of Creutzfeldt-Jacob Disease. J Clin Microbiol, JCM. 2016;54(7):1751-4.

7. WHO and European Creutzfeldt-Jakob Disease Surveillance Network criteria, as referenced and posted on the website of the UK national CJD research and surveillance unit (NCJDRSU). Available at: https://www.cjd.ed.ac.uk/sites/default/files/criteria.pdf, accessed May 6, 2020.

8. McGuire LI, Peden AH, Orru CD, et al. Real time quaking-induced conversion analysis of cerebrospinal fluid in sporadic CreutzfeldtJakob disease. Ann Neurol. 2012;72(2):278-85. doi: 10.1002/ ana.23589.

9. Rudge P, Hyare H, Green A, Collinge J, Mead S. Imaging and CSF analyses effectively distinguish CJD from its mimics. J Neurol Neurosurg Psychiatry. 2018;89(5):461-6. 